\title{
Cobertura de la disposición de excretas en Costa Rica en el periodo 2000-2014 y expectativas para el 2021
}

Excreta disposal coverage in Costa Rica from 2000 to 2014 and outlook for 2021

Darner A. Mora-Alvarado', Carlos F. Portuguez-Barquero²

Fecha de recepción: 23 de junio del 2015

Fecha de aprobación: 28 de setiembre del 2015

Mora-Alvarado, D; Portuguez-Barquero, C. Cobertura de la disposición de excretas en Costa Rica para el periodo 20002014 y expectativas en el 2021. Tecnología en Marcha. Vol. 29, № 2, Abril-Junio 2016. Pág 43-62. 


\section{Palabras clave}

Cobertura; disposición; evolución; excretas; exposición.

\section{Resumen}

El objetivo del presente estudio fue determinar las coberturas y evolución del porcentaje de población con sistemas de disposición de excretas (DE) en Costa Rica en el periodo 20002014, según los diferentes mecanismos de evacuación como alcantarillado, tanque séptico, letrinas y otros, aunado al inventario y evaluación de la remoción de la Demanda Bioquímica de Oxígeno Total (DBO mg/L) y de Coliformes fecales (CF/100 mL), en los sistemas de tratamiento convencionales de aguas residuales a cargo del AyA y el análisis de las expectativas de cobertura con DE en el país para el año 2021.

Para cumplir con el objetivo del estudio se aplicaron los siguientes pasos; primero, se analizaron las enfermedades vinculadas al contacto con las excretas; segundo, se definió el concepto de la UNICEF y la OMS de "Instalaciones de Saneamiento Mejoradas"; tercero, se describió la cobertura de la población del país con DE mediante alcantarillado, tanques sépticos, letrinas y otros, tanto a nivel nacional como regional y en la zona rural y urbana, en el año 2014; cuarto, se realizó un inventario de los sistemas de tratamiento convencional de aguas residuales y las eficiencias con la remoción de DBO total y CF/100 mL en las aguas crudas y tratadas controladas por el Laboratorio Nacional de Aguas, en el mismo año 2014; quinto, se realizó un análisis de la evolución de las coberturas de DE con los diferentes mecanismos de evacuación durante el periodo 2000-2014; por último, como sexto punto, se establecieron las expectativas de DE por alcantarillado y tanques sépticos para el año 2021.

Se hizo un resumen de los factores vinculados a la transmisión de enfermedades infecciosas transmitidas a los seres humanos por contacto con excretas. Posteriormente, se definió el concepto de "Instalaciones de Saneamiento Mejoradas" de la UNICEF y la OMS. Con el desarrollo de los datos de cobertura de la población con DE en el 2014, se comprobó que el país cuenta con un $71,5 \%$ de habitantes con tanques sépticos, $26,6 \%$ con alcantarillado, 1,5\% con letrinas y $0,4 \%$ no cuenta con ningún mecanismo de DE. Se elaboró un inventario de 58 sistemas de tratamiento de aguas residuales. Se determinaron las eficiencias de remoción de DBO total (mg/L) y CF/100 mL, comprobándose que solamente la laguna de estabilización de Santa Cruz cumple con el valor permitido de $50 \mathrm{mg} / \mathrm{L}$ en los efluentes tratados. En el caso de los sistemas de tratamiento convencionales, en su mayoría (78\%) cumplieron con el valor permitido de DBO total establecido en la normativa nacional del 2010. Con respecto a la remoción de CF/100 mL, sin bien es cierto los porcentajes son altos (entre 90\% y 100\%), las elevadas densidades provocan un gran riesgo de contaminación en los cuerpos receptores utilizados en cada sistema. El análisis de la evolución del uso de los diferentes mecanismos de DE, en el periodo de estudio, demuestra un incremento en el uso de tanques sépticos en detrimento del uso de alcantarillado hasta el 2011; no obstante, a partir de 2012 se observa una leve reversión, y en 2014 los datos indican que el uso de tanque séptico bajó a 71,5\%.

Por último, el análisis de la ampliación del alcantarillado y tratamiento en los 11 cantones de San José permitirá alcanzar entre el $22 \%$ y el $25 \%$ de población con DE tratadas y un $50 \%$ a $53 \%$ en el uso de alcantarillado. Este avance permitirá disminuir el uso de los tanques sépticos al $50 \%$ en el año 2021.

\section{Conclusiones}

De conformidad con el concepto de "Instalaciones de Saneamiento Mejoradas" de la UNICEF y la OMS, Costa Rica alcanzó un 99\% de cobertura con DE mediante alcantarillado, tanques 
sépticos y letrinas, lo que ubica al país a la vanguardia de los países latinoamericanos. Sin embargo, esto es paradójico, debido a que el poco tratamiento de las aguas residuales con sistemas colectivos, o alcantarillado, ha provocado un gran deterioro de los ríos en las zonas urbanas.

\title{
Recomendaciones
}

Es fundamental ampliar las coberturas de DE por alcantarillado sanitario con tratamiento, para sanear los ríos y otros cuerpos de agua superficiales y subterráneas; además, se debe implementar un Programa Nacional de Manejo Adecuado de las Aguas Residuales, planteando metas a mediano y largo plazo, con el propósito de mejorar la salud pública del país.

\section{Keywords}

Excreta disposal coverage; expectations; excreta evacuation.

\begin{abstract}
The present study aimed to establish excreta disposal coverage and progress in Costa Rica from 2000 to 2014, considering the different mechanisms for excreta evacuation (e.g. drainage, septic tank, and latrine), and assessing the removal of the Biochemical Oxygen Demand (BOD mg/L) and faecal coliforms $(\mathrm{CF} / 100 \mathrm{~mL})$ within conventional wastewater treatment systems managed by AyA. Likewise, the study aimed to assess the excreta disposal coverage expectations for 2021.
\end{abstract}

Six steps were followed: 1) diseases linked to excreta transmission were analysed; 2) the concept of 'Improved sanitation facilities' established by the UNICEF and the WHO was defined; 3) the excreta disposal coverage was described according to the use of drainage, septic tanks, and latrines, in a centralise and decentralise level, and in urban or rural area during 2014; 4) a stock of wastewater conventional treatment systems were carried out together with the efficiency of BOD and faecal coliforms removal in wastewater and treated water controlled by the National Water Laboratory (LNA) in 2014; 5) an assessment of the progress of excreta disposal coverage along the different mechanisms for evacuation was carried out during the period 2000-2014; and 6 ) the expectations of excreta disposal by septic tanks or drainage were established for 2021.

A summary was writing up concerning the factors linked to infectious disease transmission to humans by excreta contact. Subsequently, the concept of 'Improved sanitation facilities' established by the UNICEF and the WHO was defined. Based on the data development of the population with excreta disposal coverage in 2014, the study confirmed that $71,5 \%$ of the costarican population possesses septic tanks, 26,6 \% drainage, 1,5\% latrines, and 0,4\% lacks of any excreta evacuation mechanism. The stock presented 56 wastewater conventional treatment systems. Efficiency of BOD ( $\mathrm{mg} / \mathrm{L})$ and coliforms faecal $(\mathrm{CF} / 100 \mathrm{~mL})$ removal were determined; none of the waste stabilization ponds complied with the maximum limit value (50 $\mathrm{mg} / \mathrm{L})$. Only $57 \%$ of the 14 activated sludge treatment systems complied with the BOD allowed value established on the national normative in 2010. Coliform faecal removal percentages were high (90\% - $100 \%$ ); although, increased density of microorganisms can cause high risk of water sources pollution. The analysis of the progress of the different excreta disposal mechanisms during 2000-2014 showed a rise in the use of septic tanks, during 2000-2011. In 2012 a low reversion was observed, and in 2014 the use of septic tank was reduced to $71,5 \%$.

Finally, an analysis of the enlargement of the drainage and water treatment within 11 municipalities in San José will allow to reach $22-25 \%$ of the population with treated excreta disposal, and 
$50-53 \%$ in the use of drainage. This will allow the reduction of the use of septic tanks to $50 \%$ for 2021.

\section{Conclusions}

In accordance with the concept of 'Improved sanitation facilities', Costa Rica have reached 99 $\%$ of excreta disposal coverage due to the use of drainage, septic tanks and latrines. However, wastewater has received very little treatment (drainage and collective systems), causing great damage to the rivers within urban areas.

\section{Recommendations}

It is crucial to enlarge excreta disposal coverage, increasing drainage and wastewater treatment, in order to clean up rivers and others superficial water bodies and aquifers. A National Programme for Suitable Wastewater Manager should be implemented contemplating medium and long term goals, in order to improve public health in Costa Rica.

\section{Cobertura de la disposición de excretas en Costa Rica periodo 2000-2014 y expectativas para el 2021}

\section{Introducción}

Los seres humanos han utilizado tradicionalmente varios mecanismos para disponer sus excretas (heces y orina); el más antiguo ha sido a "cielo abierto", es decir, su inodoro fue y es la madre naturaleza. En la época en que los seres humanos eran nómadas, hacían sus necesidades fisiológicas cerca de los árboles y ríos. Pero cuando decidieron vivir en un solo sitio, tuvieron la necesidad de deshacerse de sus excretas "tan lejos como les pidiera su nariz y tan cerca como lo exigiera su sistema digestivo" (Mora, 2005). En razón de esto surgieron cuatro mecanismos, a saber, fosas sépticas o letrinas, tanques sépticos, alcantarillado (sin tratamiento o cloacas) y alcantarillado sanitario (AS) (AyA, 2009). Estos últimos tienen en común el uso del inodoro, que se originó en Creta hace más de 4000 años. Los egipcios y los romanos también avanzaron en el uso de inodoros, mediante la utilización de baños públicos cuyo símbolo histórico es la conocida "Cloaca Máxima de Roma" (Pickover, 2012); sin embargo, fueron clausurados porque fomentaban la promiscuidad sexual, lo que provocó un retroceso en el saneamiento de las comunidades. Es así como, en el periodo comprendido entre los siglos $\mathrm{V}$ y XV, las calles estaban llenas de excrementos. Los humanos defecaban en las esquinas, ríos y árboles, y en el mejor de los casos usaban la bacenilla, cuyo contenido iba a parar a la calle tras el grito “iAgua va!”. Luego, en 1589, el inglés John Hannington desarrolló el retrete de válvula, que fue instalado en el palacio de Isabel I. En 1668, el Comisionado de Paris ordenó la instalación de inodoros en todas las casas y en 1840 el Acta de Salud Pública Inglesa obligó a instalar inodoros en toda Europa (Llopie \& Tynan, 2011). En esta época se incrementaron las mejoras en los servicios de agua y alcantarillado, pero fue hasta principios del siglo XX que se fomentó la recolección de aguas residuales domésticas o excretas y el tratamiento, antes de descargarlas en un cuerpo receptor (río, quebrada o mar) (Artman \& Chadwick, 2015).

En Costa Rica, las primeras obras de alcantarillado se inauguraron en 1911, y posteriormente se construyeron en las ciudades de Alajuela, Heredia y Cartago, a principios de la década de 1940 (Araya, 1992). Luego, se construyó el sistema de tratamiento mediante lagunas de estabilización en Cañas (1959), Guanacaste. Lamentablemente, las plantas de tratamiento del Valle Central fueron abandonadas a partir de 1963 (Mora, 1992), aumentando así la contaminación orgánica de los ríos de la Cuenca Virilla-Tárcoles (Cuenca 24). Sin embargo, gracias a un convenio entre el Gobierno y el Instituto Costarricense de Acueductos y Alcantarillados (AyA) (1972-1975), 
se construyeron lagunas facultativas en las ciudades de Liberia, San Isidro de Pérez Zeledón, Nicoya y Santa Cruz, y en 2004 se inauguró el Emisario Submarino de Limón (Araya, Barboza, Ramírez \& Rodríguez, 2009).

Pero aún con estas obras Costa Rica ocupa uno de los últimos lugares en el tratamiento con sistemas convencionales en América Latina (Reynolds, 2015). De acuerdo con el Programa Conjunto de Monitoreo del Fondo para la Infancia de las Naciones Unidas (UNICEF) y la Organización Mundial de la Salud (OMS), con el concepto "Instrumentos de Saneamiento Mejorados", Costa Rica ocupa uno de los primeros lugares en esta misma zona. Esto se debe a que el concepto abarca la disposición de excretas con alcantarillado sanitario, alcantarillado sin tratamiento, tanques sépticos y letrinas (UNICEF/OMS, 2006).

El Laboratorio Central del AyA, hoy Laboratorio Nacional de Aguas (LNA), mediante el Decreto Ejecutivo 26066-S (Poder Ejecutivo-Costa Rica, 1997), elabora informes anuales de calidad del agua para consumo humano y disposición de excretas desde 1991 (Mora, 2009). Sin embargo, en este año, con los datos al 2014 se decidió separar ambos temas, con el propósito de brindar el peso específico que tiene el suministro de agua y la disposición adecuada de excretas (DAE) o saneamiento como determinantes de la salud pública (Baltazar \& Solon, 1989; Heller, 1997). En razón de esto, el presente informe se enfoca en la evaluación y evolución de las coberturas en la DAE desde el año 2000 hasta el 2014, mediante los mecanismos de alcantarillado sanitario, alcantarillado sin tratamiento, tanques sépticos (TS) y letrinas o fosas sépticas (excusado de hueco) en Costa Rica en los últimos 15 años (2000-2014).

\section{Objetivos}

\section{General}

Determinar las coberturas y evolución de población con disposición de excretas (DE) en Costa Rica en el periodo 2000-2014, según los diferentes mecanismos de evacuación, tales como alcantarillado, tanque séptico, letrinas y otros, aunado al inventario y evaluación de la remoción de la Demanda Bioquímica de Oxígeno total (DBO)(mg/L) y de Coliformes fecales (CF/100 $\mathrm{mL}$ ), en los sistemas de tratamiento convencionales de aguas residuales a cargo del AyA, y el análisis de las expectativas en las coberturas de DE en el país para el año 2021.

\section{Específicos}

- Describir la clasificación de las infecciones relacionadas al contacto humano con excretas a nivel mundial.

- Describir el concepto de "Instalaciones de Saneamiento Mejoradas" (ISM) de la UNICEF y la OMS.

- Describir la cobertura de la población mediante los mecanismos de alcantarillado, tanques sépticos, letrinas y otros en el país, y su distribución por regiones en el año 2014.

- A nivel nacional, inventariar y evaluar la remoción de DBO y Coliformes fecales en los sistemas de tratamiento de aguas residuales, analizadas por el LNA en 2014.

- Recopilar y analizar información comparativa de las coberturas de DE O ISM (alcantarillado, tanques sépticos, letrinas y otros), en el periodo 2000-2014 en Costa Rica).

- Establecer las expectativas de cobertura por alcantarillado, tanques sépticos y letrinas, con la implementación del proyecto "Mejoramiento Ambiental de San José, al año 2021". 


\section{Metodología}

Para cumplir con los objetivos de este estudio descriptivo se aplicaron los siguientes pasos:

Enfermedades vinculadas al contacto con las excretas

La identificación de las enfermedades vinculadas al contacto con las excretas se realizó con la Clasificación de Infecciones Relacionadas con las Excretas, descrito en el libro Saneamiento, Educación y Salud, de Mora (2005).

Concepto de Instalaciones de Saneamiento Mejoradas

La descripción del concepto de ISM fue definida por la UNICEF y la OMS en el documento Progreso para la Infancia: un balance sobre Agua y Saneamiento.

Cobertura de población según mecanismo de DE o ISM en el año 2014

Las coberturas de población, por país y regiones de planificación de Costa Rica, se obtuvieron de los datos de la Encuesta Nacional de Hogares del año 2014 (INEC, 2014).

Sistemas de tratamiento convencional de aguas residuales en Costa Rica

Se realizó un inventario de los sistemas de tratamiento de aguas residuales ordinarias, a nivel nacional, con datos del AyA y el Decimonoveno Informe Estado de la Nación en Desarrollo Humano Sostenible (Programa Estado de la Nación, 2013).

La identificación de los sistemas de tratamiento de aguas residuales, cubiertas por el LNA, y la remoción de DBO y Coliformes fecales, se obtuvo del Informe Anual de Aguas Residuales. Para efectos prácticos, si el lector requiere analizar los otros datos de Sólidos Totales (ST), DQO (mg/L), Grasas (mg/L), Nitrógeno total $(\mathrm{N})$, Fósforo total (P) y otros, los autores recomiendan utilizar el Informe Anual de Aguas Residuales del 2014 del LNA (Ramírez \& Valiente, 2015).

Análisis de la evolución de coberturas de población con disposición de excretas 2000-2014

La evolución de las coberturas de población con disposición de excretas mediante alcantarillado, TS y letrinas durante el periodo 2000-2014 se elaboró con los datos de las encuestas de hogares anuales de esos años y los censos 2000 y 2011 (INEC, 2001, 2011).

Expectativas de saneamiento para el año 2021

Las expectativas de DE para el año 2021 se establecieron con los siguientes documentos:

- Proyecto de Mejoramiento Ambiental de San José (Unidad Ejecutora AyA-JBIC, 2015; Navarro, 2015).

- Programa Nacional de Manejo Adecuado de las Aguas Residuales (AyA, 2009).

- Agua para consumo humano y disposición de excretas en Costa Rica: situación actual y expectativa (Mora, 2003).

Resultados

Enfermedades infecciosas relacionadas con el contacto con excretas

Los factores que influyen en la transmisión de enfermedades mediante el contacto con excretas se resumen en el siguiente esquema: 


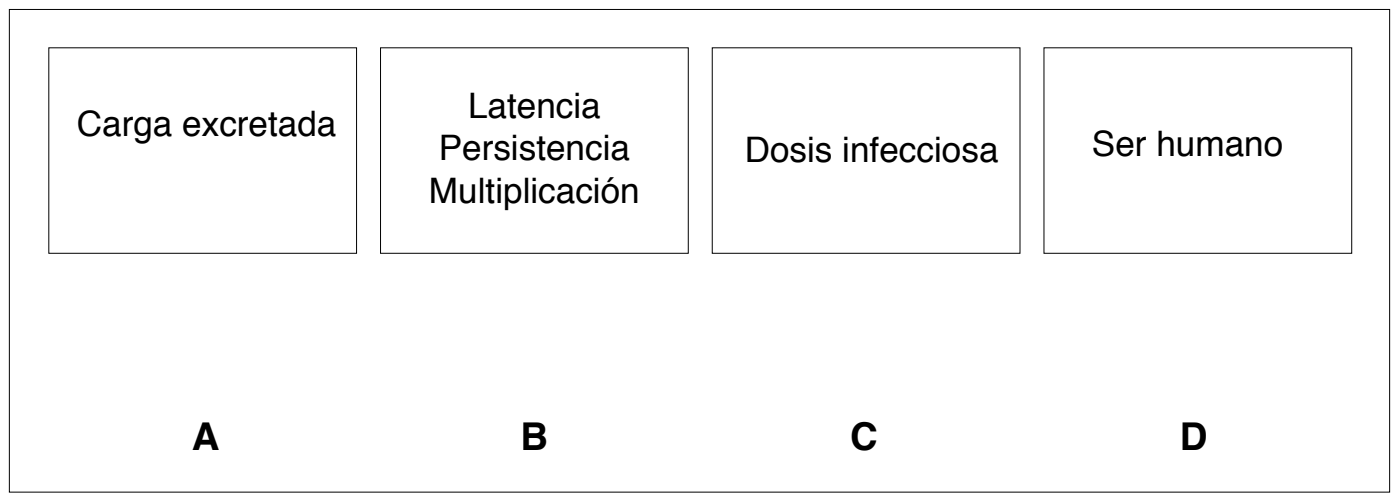

Fuente: Los servicios de agua potable y saneamiento en el umbral del siglo XXI (Jouravlev, A, 2004).

a) La carga excretada dependerá del estado de salud de la persona y del contenido de microbios patógenos en sus heces.

b) La latencia, persistencia y multiplicación se refiere al tiempo que pueden sobrevivir los microorganismos patógenos, su resistencia al ambiente y su multiplicación en la excreta o el suelo.

c) La dosis infecciosa dependerá del tipo de microorganismo; por ejemplo, los virus y bacterias necesitan una mayor dosis infecciosa que los protozoarios y helmintos.

d) La enfermedad en el ser humano dependerá del estado de su aparato inmunológico y de la patogeneidad del microorganismo infectante.

Cuadro 1. Clasificación ambiental de las infecciones relacionadas con las excretas

\begin{tabular}{|c|c|c|c|c|}
\hline Categoría & $\begin{array}{l}\text { Característica } \\
\text { epidemiológica }\end{array}$ & Infección & $\begin{array}{l}\text { Vía dominante } \\
\text { de transmisión }\end{array}$ & $\begin{array}{l}\text { Principales medidas de } \\
\text { control }\end{array}$ \\
\hline $\begin{array}{l}\text { Enfermedades } \\
\text { fecal-orales no } \\
\text { bacterianas }\end{array}$ & $\begin{array}{l}\text { No latentes, } \\
\text { baja dosis } \\
\text { infecciosa. }\end{array}$ & $\begin{array}{l}\text { Enterobiasis, } \\
\text { Infeciones por } \\
\text { enterovirus, } \\
\text { Himenolepsiasis, } \\
\text { Amibiasis, } \\
\text { Giardiasis, } \\
\text { Balantidiasis }\end{array}$ & $\begin{array}{l}\text { Personal, } \\
\text { doméstica }\end{array}$ & $\begin{array}{l}\text { Abastecimiento } \\
\text { doméstico de agua, } \\
\text { educación sanitaria, } \\
\text { mejora de viviendas, } \\
\text { instalación de letrinas }\end{array}$ \\
\hline $\begin{array}{l}\text { Enfermedades } \\
\text { fecal-orales } \\
\text { bacterianas }\end{array}$ & $\begin{array}{l}\text { No latentes, } \\
\text { media o alta } \\
\text { dosis infecciosa; } \\
\text { moderadamente } \\
\text { persistentes, sin } \\
\text { huésped }\end{array}$ & $\begin{array}{l}\text { Fiebre, tifoidea } \\
\text { y paratifoidea, } \\
\text { Salmonelosis, } \\
\text { disentería, bacilar, } \\
\text { cólera, diarrea por } \\
\text { E. coli, enteritis por } \\
\text { Campylobacter }\end{array}$ & $\begin{array}{l}\text { Personal, } \\
\text { doméstica, } \\
\text { agua, } \\
\text { alimentos }\end{array}$ & $\begin{array}{l}\text { Abastecimiento } \\
\text { doméstico de agua, } \\
\text { educación sanitaria, } \\
\text { mejora de viviendas, } \\
\text { instalación de letrinas, } \\
\text { tratamiento de las } \\
\text { excretas antes de la } \\
\text { disposición o reúso }\end{array}$ \\
\hline $\begin{array}{l}\text { Helmintos del } \\
\text { suelo }\end{array}$ & $\begin{array}{l}\text { Latentes, } \\
\text { persistentes, } \\
\text { sin huésped } \\
\text { intermediario }\end{array}$ & $\begin{array}{l}\text { Ascariasis, } \\
\text { Tricuriasis y } \\
\text { Anquilostomiasis }\end{array}$ & $\begin{array}{l}\text { Jardín, } \\
\text { campos y } \\
\text { agricultura }\end{array}$ & $\begin{array}{l}\text { Instalación de letrinas, } \\
\text { tratamiento de excretas } \\
\text { antes de la aplicación en } \\
\text { el terreno }\end{array}$ \\
\hline
\end{tabular}




\begin{tabular}{|c|c|c|c|c|}
\hline Categoría & $\begin{array}{l}\text { Característica } \\
\text { epidemiológica }\end{array}$ & Infección & $\begin{array}{l}\text { Vía dominante } \\
\text { de transmisión }\end{array}$ & $\begin{array}{l}\text { Principales medidas de } \\
\text { control }\end{array}$ \\
\hline Teniasis & $\begin{array}{l}\text { Latentes, } \\
\text { persistentes, } \\
\text { con huésped } \\
\text { intermediario }\end{array}$ & Teniasis & $\begin{array}{l}\text { Jardín, } \\
\text { campos y } \\
\text { pastizales }\end{array}$ & $\begin{array}{l}\text { Instalación de letrinas, } \\
\text { tratamiento de excretas } \\
\text { antes de la aplicación } \\
\text { en el terreno, cocción, } \\
\text { inspección de la carne }\end{array}$ \\
\hline $\begin{array}{l}\text { Helmintos del } \\
\text { agua }\end{array}$ & $\begin{array}{l}\text { Latentes, } \\
\text { persistentes, } \\
\text { con huésped } \\
\text { intermediario }\end{array}$ & $\begin{array}{l}\text { Esquistosomiasis } \\
\text { y otras } \\
\text { enfermedades } \\
\text { provocadas por } \\
\text { helmintos }\end{array}$ & Agua & $\begin{array}{l}\text { Instalación de letrinas, } \\
\text { tratamiento de excretas } \\
\text { antes de la disposición } \\
\text { en el agua, control del } \\
\text { reservorio animal }\end{array}$ \\
\hline $\begin{array}{l}\text { Enfermedades } \\
\text { transmitidas por } \\
\text { insectos }\end{array}$ & $\begin{array}{l}\text { Insectos } \\
\text { vectores } \\
\text { relacionados } \\
\text { con las excretas }\end{array}$ & $\begin{array}{l}\text { Filariasis y todas } \\
\text { las infecciones } \\
\text { mencionadas en } \\
\text { las categorías } 1 \\
\text { a } 5 \text {, en donde } \\
\text { las moscas y } \\
\text { las cucarachas } \\
\text { pueden ser } \\
\text { vectores }\end{array}$ & $\begin{array}{l}\text { Varios lugares } \\
\text { contaminados } \\
\text { por heces, } \\
\text { en donde se } \\
\text { reproducen los } \\
\text { insectos }\end{array}$ & $\begin{array}{l}\text { Identificación y } \\
\text { eliminación de los } \\
\text { lugares adecuados para } \\
\text { la reproducción }\end{array}$ \\
\hline
\end{tabular}

Fuente: Feachem et al. (1983a).

\section{Concepto de Instalaciones de Saneamiento Mejoradas (ISM)}

De conformidad con las metas establecidas en los Objetivos de Desarrollo del Milenio (ODM), del Programa Conjunto de Monitoreo (PCM) de la UNICEF y la OMS, las ISM se definen de la siguiente manera:

a) Mejoradas

- Letrina de sifón con descarga.

- Una cloaca con tubería.

- Un tanque séptico.

- Letrina de pozo mejorada con ventilación.

- Letrina con pozo con loza.

- Retrete de compostaje.

b) No mejoradas

- Letrina con sifón que descarga en otra parte.

- Letrina de pozo sin losa/pozo abierto.

- Balde o cubo.

- Retrete colgante/letrina colgante.

- A cielo abierto. 
Cobertura de población con disposición de excretas

La cobertura de la población por zona urbana, rural y regiones de planificación en el año 2014 se presenta en el Cuadro 2.

Cuadro 2. Costa Rica y regiones de planificación: población con servicio sanitario y tipo de disposición de excretas, 2014

\begin{tabular}{|c|c|c|c|c|}
\hline Poblaciones & $\begin{array}{c}\text { Conectado a tanque } \\
\text { séptico }\end{array}$ & $\begin{array}{c}\text { Conectado a } \\
\text { alcantarillado o cloaca }\end{array}$ & Letrina y otros & No tienen \\
\hline Total 4.772 .098 & $3.412 .099(71,5 \%)$ & $1.268 .705(26,6 \%)$ & $73.462(1,5 \%)$ & $17.832(0,4 \%)$ \\
\hline Zona & & & & \\
Urbana: 3.469 .802 & $2.269 .324(65,4 \%)$ & $1.175 .479(51,8 \%)$ & $13.653(0,4 \%)$ & $11.346(0,32 \%)$ \\
Rural : 1.302.296 & $1.142 .775(87,75)$ & $93.226(7,2 \%)$ & $58.809(5,14 \%)$ & $6.486(0,50 \%)$ \\
\hline Región & & & & \\
Central: 2.975 .481 & $1.912 .640(64,3 \%)$ & $1.041 .467(35 \%)$ & $13.235(0,46 \%)$ & $8.139(0,27 \%)$ \\
Chorotega: 359.371 & $301.137(83,8 \%)$ & $39.307(10,9 \%)$ & $17.552(4,8 \%)$ & $1.375(0,4 \%)$ \\
P. Central: 275.483 & $214.698(78,2 \%)$ & $56.545(20,6 \%)$ & $3.658(1,3 \%)$ & $552(0,2 \%)$ \\
Brunca: 356.519 & $317.730(87,4 \%)$ & $33.485(9,4 \%)$ & $10.365(2,9 \%)$ & $939(0,26 \%)$ \\
H. Atlántica: 428.133 & $341.101(79,8 \%)$ & $69.258(16,2 \%)$ & $14.194(3,3 \%)$ & $3.580(0,84 \%)$ \\
H. Norte:377.111 & $330.793(87,6 \%)$ & $28.643(7,6 \%)$ & $14.458(3,8 \%)$ & $3.217(0,85 \%)$ \\
\hline
\end{tabular}

Fuente: Encuesta de Hogares 2014 y LNA.

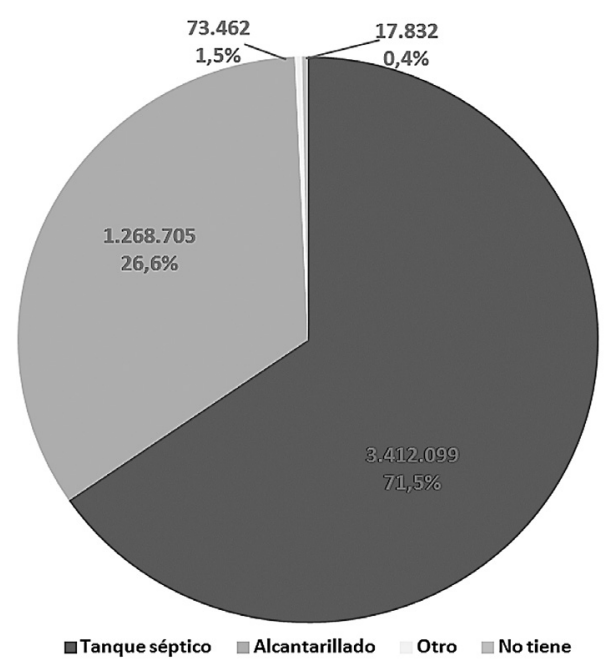

Inventario de sistemas de tratamiento de aguas residuales

Se presenta el inventario del LNA, mediante el Área de Aguas Residuales, con los siguientes sistemas de tratamiento de aguas residuales domésticos: 
Cuadro 3. Sistemas de tratamiento de aguas residuales operados por el AyA

\begin{tabular}{|c|c|c|c|c|}
\hline \multicolumn{5}{|c|}{ Sistemas periféricos de tratamiento de aguas residuales del AyA } \\
\hline Lugar & $\begin{array}{c}\text { Año de } \\
\text { construcción }\end{array}$ & Características & Tipo & $\begin{array}{l}\text { Cumple el decreto } \\
\text { 33601-MINAE-S }\end{array}$ \\
\hline Liberia & 1974 & $\begin{array}{c}\text { Remodelada en } \\
2011\end{array}$ & Laguna facultativa & No \\
\hline Cañas & 1974 & $\begin{array}{l}\text { Separa aguas } \\
\text { pluviales de } \\
\text { residuales }\end{array}$ & Laguna facultativa & No \\
\hline Nicoya & 1974 & En estudio & Laguna facultativa & No \\
\hline Santa Cruz & 1974 & $\begin{array}{l}\text { Con capacidad para } \\
\text { recibir más agua }\end{array}$ & Laguna facultativa & Sí \\
\hline $\begin{array}{c}\text { San Isidro de Pérez } \\
\text { Zeledón }\end{array}$ & 1974 & Dos plantas & Laguna facultativa & No \\
\hline $\begin{array}{c}\text { Boruca, Buenos Aires } \\
\text { de Puntarenas }\end{array}$ & 1980 & Donada por Pindeco & Laguna facultativa & No \\
\hline El Roble, Puntarenas & 1974 & $\begin{array}{l}\text { La más grande del } \\
\text { país }\end{array}$ & Lodos convencionales & No \\
\hline Limón & 2005 & $\begin{array}{c}\text { Con capacidad } \\
\text { para cinco veces lo } \\
\text { actual }\end{array}$ & Emisario submarino & Sí \\
\hline Los Reyes, Alajuela & 2005 & & Lodos activados & Sí \\
\hline El Coyol, Alajuela & & & FAFA ${ }^{1}$ & Sí \\
\hline Santa Cecilia Puriscal & & Filtro anaerobio* & RAFA $^{2}$ & Sí \\
\hline Bosques de Santa Ana & 2000 & & RAFA & No \\
\hline Bulevar Las Palmas & 2003 & & $\begin{array}{l}\text { Lodos activados con } \\
\text { aireación ext. }\end{array}$ & Sí \\
\hline Rincón Verde II & 2001 & $\begin{array}{c}\text { Mejora al sistema } \\
\text { electromecánico y } \\
\text { sedimentador } 2011 \text { - } \\
2012\end{array}$ & $\begin{array}{l}\text { Lodos activados } \\
\text { aireación ext. }\end{array}$ & Sí \\
\hline Monte Lindo & 2003 & & & Sí \\
\hline André Chalé & 2000 & $\begin{array}{l}\text { Rehabilitación del } \\
\text { sistema } 2011\end{array}$ & & Sí \\
\hline $\begin{array}{l}\text { Villa Verano de } \\
\text { Alajuela }\end{array}$ & & & & Sí \\
\hline Lomas Pindeco & & & & Sí \\
\hline Lomas de Zurquí & & & & No \\
\hline $\begin{array}{l}\text { Santa Isabel de } \\
\text { Heredia }\end{array}$ & 2010 & & & No \\
\hline Don Edwin-Guápiles & & & & Sí \\
\hline
\end{tabular}

Fuente: Laboratorio Nacional de Aguas (2014).

1 FAFA: filtro anaerobio de flujo ascendente.

2 RAFA: reactor anaerobio de flujo ascendente.

*Santa Cecilia de Puriscal tiene un filtro biológico al final del tratamiento. 
Cuadro 3A. Sistemas de tratamiento de aguas residuales operados por la Empresa de Servicios Públicos de Heredia (ESPH)

\begin{tabular}{|c|c|c|c|c|}
\hline \multicolumn{5}{|c|}{ Sistemas operados por la ESPH } \\
\hline Lugar & $\begin{array}{c}\text { Año de } \\
\text { construcción }\end{array}$ & Características & Tipo & $\begin{array}{c}\text { Cumple el decreto } \\
\text { 33601-MINAE-S }\end{array}$ \\
\hline Los Lagos & 1975 & Carrusel & Lodos activados & Sí \\
\hline La Aurora & 1977 & Remodelada & Lodos activados & Sí \\
\hline Real Santamaría Este & 2003 & Remodelada & $\begin{array}{c}\text { Lodos activados } \\
\text { aireación ext. } \\
\text { Lodos activados } \\
\text { aireación ext. }\end{array}$ & Sí \\
\hline Real Santamaría Oeste & 2003 & Utiliza lirios & Laguna de oxidación & No \\
\hline Las Flores & 1999 & &
\end{tabular}

Fuente: ESPH (2013).

Cuadro 3B. Sistemas de tratamiento operados por municipalidades y ASADAS

\begin{tabular}{|c|c|c|}
\hline Lugar & Cantidad & Tipo \\
\hline M. Belén & 4 & Anaerobias \\
\hline M. Alajuela & 10 & Aerobi/anae \\
\hline M. Flores & 1 & Aerobia \\
\hline M. Cartago & 5 & Aerob/anae \\
\hline M. Escazú & 2 & ND \\
\hline A Lomas de Zurquí & 1 & Aerobia \\
\hline A Paso de las Garzas & 1 & Aerobia \\
\hline A Orosi, Cartago & 1 & Anaerobia \\
\hline A Herediana, Siquirres & 1 & Aerobia \\
\hline A Venecia Matina & 1 & Aerobia \\
\hline A Cariblanco, Sarapiquí & 1 & Aerobia \\
\hline A Horquetas, Sarapiqui & 1 & Anaerobia \\
\hline A Limón 2000 & 1 & Laguna est. \\
\hline A Mora de Turrialba & 1 & Anaerobia \\
\hline A Carmen Lyra, Turrialba & 1 & Anaerobia \\
\hline
\end{tabular}

Fuente: Elaboración propia con base en datos de Focard-APS (Ruiz, 2012).

En total, el número de sistemas de tratamiento de aguas residuales domésticos en funcionamiento es de 58. 
Remoción de DBO total y coliformes fecales en los sistemas de tratamiento

En el cuadro 3 se presenta la remoción de DBO total (mg/L) y CF/100 mL, en los sistemas de tratamiento de aguas residuales domésticos operados por el AyA, en el periodo 2014.

Cuadro 4. Remoción de DBO total y Coliformes fecales en los sistemas de tratamiento operados por el AyA

\begin{tabular}{|c|c|c|c|c|c|c|}
\hline Variables & $\begin{array}{c}\text { Tipos de } \\
\text { agua }\end{array}$ & $\begin{array}{l}\text { Laguna } \\
\text { Cañas }\end{array}$ & Laguna Liberia & $\begin{array}{c}\text { Laguna Santa } \\
\text { Cruz }\end{array}$ & $\begin{array}{l}\text { Laguna } \\
\text { Nicoya }\end{array}$ & $\begin{array}{c}\text { Laguna Pérez } \\
\text { Zeledón }\end{array}$ \\
\hline \multirow{3}{*}{ DBO mg/L } & Cruda & 278 & 135 & 261 & 284 & 303 \\
\hline & Tratada & 91 & 58 & 86 & 100 & 77 \\
\hline & $\%$ eficiencia & $67 \%$ & $57 \%$ & $67 \%$ & $65 \%$ & $75 \%$ \\
\hline \multirow{3}{*}{$\begin{array}{c}\text { Coliformes } \\
\text { fecales } \\
100 \mathrm{~mL}\end{array}$} & Cruda & 56.362 .604 & 41.968 .360 & 9.110 .785 & 13.684 .248 & 13.379 .234 \\
\hline & Tratada & 67.585 & 229.638 & 155.483 & 1.282 .430 & 304.510 \\
\hline & $\%$ eficiencia & $100 \%$ & $99 \%$ & $98 \%$ & $91 \%$ & $98 \%$ \\
\hline Variables & $\begin{array}{l}\text { Tipos de } \\
\text { agua }\end{array}$ & $\begin{array}{c}\text { Sistema } \\
\text { tratamiento } \\
\text { El Roble de } \\
\text { Puntarenas }\end{array}$ & $\begin{array}{c}\text { Sistema } \\
\text { tratamiento } \\
\text { Bosques de } \\
\text { Santa Ana }\end{array}$ & $\begin{array}{c}\text { Sistema } \\
\text { tratamiento } \\
\text { Monte Lindo }\end{array}$ & $\begin{array}{c}\text { Sistema } \\
\text { tratamiento } \\
\text { Rincón Verde }\end{array}$ & $\begin{array}{c}\text { Sistema } \\
\text { tratamiento } \\
\text { André Chalé }\end{array}$ \\
\hline \multirow{3}{*}{ DBO mg/L } & Cruda & 298 & 256 & 252 & 252 & 334 \\
\hline & Tratada & 195 & 123 & $40^{*}$ & $22^{*}$ & $24^{*}$ \\
\hline & $\%$ eficiencia & $35 \%$ & $52 \%$ & $84 \%$ & $91 \%$ & $93 \%$ \\
\hline \multirow{3}{*}{$\begin{array}{l}\text { Coliformes } \\
\text { fecales } \\
100 m L\end{array}$} & Cruda & 19.535 .840 & 19.715 .268 & 2.171 .263 & 4.074 .151 & 58.029 .961 \\
\hline & Tratada & 56.651 .624 & 5.852 .605 & 395.654 & 258.854 & 97.196 \\
\hline & $\%$ eficiencia & $-190 \%$ & $70 \%$ & $82 \%$ & $94 \%$ & $100 \%$ \\
\hline Variables & $\begin{array}{c}\text { Tipos de } \\
\text { agua }\end{array}$ & $\begin{array}{c}\text { Sistema } \\
\text { tratamiento } \\
\text { Las Palmas } \\
\text { Ciudad Colón }\end{array}$ & $\begin{array}{c}\text { Sistema } \\
\text { tratamiento } \\
\text { Hacienda Los } \\
\text { Reyes }\end{array}$ & $\begin{array}{c}\text { Sistema } \\
\text { tratamiento } \\
\text { Villa Verano } \\
\text { Alajuela }\end{array}$ & $\begin{array}{c}\text { Sistema } \\
\text { tratamiento } \\
\text { Santa Cecilia } \\
\text { Puriscal }\end{array}$ & $\begin{array}{c}\text { Sistema } \\
\text { tratamiento } \\
\text { Boruca } \\
\text { Buenos Aires }\end{array}$ \\
\hline \multirow{3}{*}{ DBO mg/L } & Cruda & 199 & 166 & 176 & 360 & 321 \\
\hline & Tratada & $23^{*}$ & 99 & $35^{\star}$ & 86 & 103 \\
\hline & $\%$ eficiencia & $88 \%$ & $40 \%$ & $65 \%$ & $76 \%$ & $68 \%$ \\
\hline \multirow{3}{*}{$\begin{array}{l}\text { Coliformes } \\
\text { fecales } \\
100 \mathrm{~mL}\end{array}$} & Cruda & 7.835 .375 & 12.157 .895 & 12.128 .477 & 22.650 .773 & 10.074 .242 \\
\hline & Tratada & 157.830 & 242.378 & 981.870 & 5.832 .753 & 1.354 .287 \\
\hline & $\%$ eficiencia & $98 \%$ & $80 \%$ & $92 \%$ & $74 \%$ & $87 \%$ \\
\hline Variables & $\begin{array}{l}\text { Tipos de } \\
\text { agua }\end{array}$ & $\begin{array}{c}\text { Sistema } \\
\text { tratamiento } \\
\text { Lomas de } \\
\text { Pindeco }\end{array}$ & $\begin{array}{c}\text { Sistema } \\
\text { tratamiento } \\
\text { Lomas de } \\
\text { Zurquí }\end{array}$ & $\begin{array}{c}\text { Sistema } \\
\text { tratamiento } \\
\text { Santa Isabel } \\
\text { Heredia }\end{array}$ & $\begin{array}{c}\text { Sistema } \\
\text { tratamiento } \\
\text { Don Edwin } \\
\text { Guápiles }\end{array}$ & ----- \\
\hline \multirow{3}{*}{ DBO mg/L } & Cruda & 212 & 269 & 377 & 365 & --- \\
\hline & Tratada & $42^{*}$ & 81 & 57 & $30^{*}$ & ---- \\
\hline & \%Eficiencia & $80 \%$ & $70 \%$ & $85 \%$ & $92 \%$ & --- \\
\hline \multirow{3}{*}{$\begin{array}{l}\text { Coliformes } \\
\text { fecales } \\
\text { 100mL }\end{array}$} & Cruda & 18.342 .218 & 8.958 .243 & 13.000 .000 & 32.832 .910 & --- \\
\hline & Tratada & 16.773 & 3.124 .127 & 2.400 .000 & 269.444 & --- \\
\hline & $\%$ eficiencia & $100 \%$ & $65 \%$ & $82 \%$ & $99 \%$ & ---- \\
\hline
\end{tabular}

Fuente: Área de Aguas Residuales-LNA.

Nota: ${ }^{*}$ Cumple con la normativa de Costa Rica. 
Cuadro 5. Caracterización de la calidad del agua marina en sitios cercanos a la descarga del emisario submarino - Limón. Sitio del desfogue del emisor submarino a tres profundidades diferentes. Muestra de agua compuesta integrada a partir de tres submuestras: fondo, medio y superficie.

\begin{tabular}{|c|c|c|c|c|}
\hline & 12-feb.-14 & 30 -jul.-14 & 25 -set.-14 & 11-nov.-14 \\
\hline \multicolumn{5}{|l|}{ PARÁMETRO } \\
\hline Material flotante & Ausente & Ausente & Ausente & Ausente \\
\hline Aspecto del agua & Clara & Oscura & Clara & Clara \\
\hline Estado del tiempo & Soleado & Fresco & Soleado & Soleado \\
\hline Hora de recolección & $15: 00$ & $14: 40$ & $14: 40$ & $14: 00$ \\
\hline Temperatura ambiente, ${ }^{\circ} \mathrm{C}$ & 32,0 & 29,0 & 31,0 & 32,0 \\
\hline Temperatura agua, ${ }^{\circ} \mathrm{C}$ & 28,5 & 28,2 & 29,7 & 29,6 \\
\hline Amonio, mg/L & 0,11 & 0,20 & 0,12 & 0,29 \\
\hline $\mathrm{COT}, \mathrm{mg} / \mathrm{L}$ & & & 4,34 & \\
\hline Color verdadero, U Pt/Co & & 31 & 9,0 & 3,0 \\
\hline Conductividad, US/cm & 52400 & 51100 & 50300 & 51400 \\
\hline DBO Total,mg/L & 3,0 & 3,0 & 1,7 & 1,9 \\
\hline DQO Total,mg/L & 98 & 91 & 131 & 98 \\
\hline DQO Soluble,mg/L & 44 & 80 & 117 & 91 \\
\hline Fósforo, mg/L & 0,0 & 0,03 & 0,03 & 0,03 \\
\hline Nitratos, mg/L & 1,0 & 6,3 & 1,3 & 6,5 \\
\hline Nitróg. Am., mg/L & 0,10 & 0,16 & 0,09 & 0,22 \\
\hline Oxígeno disuelto real, $\mathrm{mg} / \mathrm{L}$ & 8,4 & 7,5 & 7,6 & 7,3 \\
\hline Oxígeno disuelto teórico, mg/L & 7,83 & 7,83 & 7,56 & 7,56 \\
\hline Porcentaje Sat. de Oxígeno Dis. & 107 & 96 & 101 & 97 \\
\hline $\mathrm{pH}$ & 7,35 & 8,10 & 8,16 & 8,03 \\
\hline S. T. T, mg/L & 43011 & 41273 & 47721 & 40710 \\
\hline S. S. T, mg/L & 20 & 36 & 21 & 12 \\
\hline S. D. T, mg/L & 42991 & 41237 & 47700 & 40698 \\
\hline S. S. S. $\mathrm{mL} / \mathrm{L} / \mathrm{hr}$ & 0 & 0 & 0 & 0 \\
\hline Turbiedad, UNT & & 26 & 17 & 7,0 \\
\hline S.A.A.M., mg/L & 0,260 & 0,200 & 0,043 & 0,170 \\
\hline \multicolumn{5}{|l|}{ Altura Secchi, metros } \\
\hline Coliformes fecales, NMP/100mL & 0 & 170 & & \\
\hline Enterococos NMP/100mL & 0 & 33 & & \\
\hline CLASIFICACIÓN & 1 & 1 & 1 & 1 \\
\hline SUMA DE PUNTOS & 3 & 3 & 3 & 3 \\
\hline CÓDIGO DE COLOR & Azul & Azul & Azul & Azul \\
\hline
\end{tabular}

Continúa... 


\begin{tabular}{|c|c|c|c|c|}
\hline & 12 -feb.-14 & 30 -jul.-14 & 25 -set.-14 & 11-nov-14 \\
\hline PARÁMETRO & & & & \\
\hline INTERPRETACIÓN DE CALIDAD & $\operatorname{Sin}$ & $\operatorname{Sin}$ & $\operatorname{Sin}$ & $\operatorname{Sin}$ \\
\hline & contaminación & contaminación & contaminación & contaminación \\
\hline
\end{tabular}

Fuente: LNA: José Miguel Ramírez y Carmen Valiente.

\section{Esquema del Emisario Submarino en Limón}

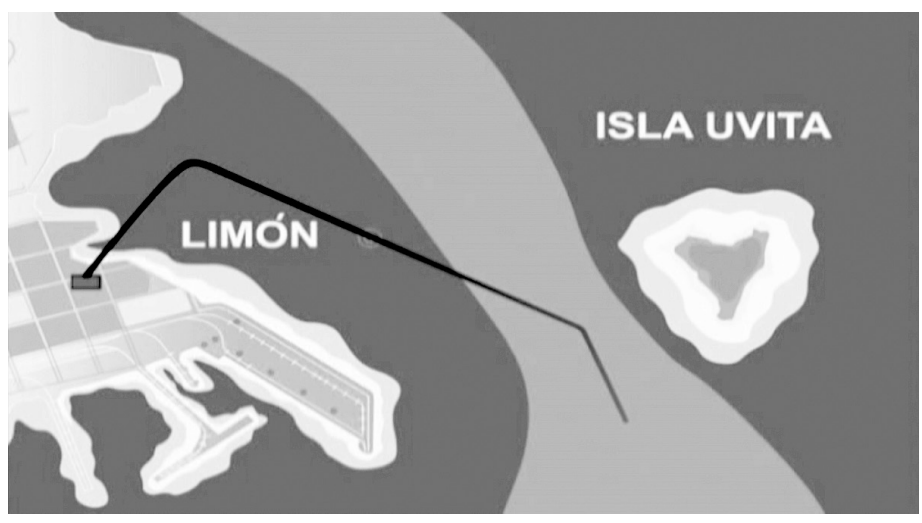

\section{Observaciones}

Muestreo compuesto. Muestra integrada por volúmenes iguales de 1 litro, para un volumen final de 3 litros de muestra compuesta. El agua fue recolectada en el fondo a $17 \mathrm{~m}$; en la parte media a 9 m y en la superficie a 10-15 cm y luego se mezcló.

Análisis comparativo o evolución de las coberturas de disposición de excretas en Costa Rica

En el siguiente gráfico se presenta el análisis comparativo o evolución de las coberturas, según el tipo de disposición de excretas en Costa Rica, periodo 2000-2014.

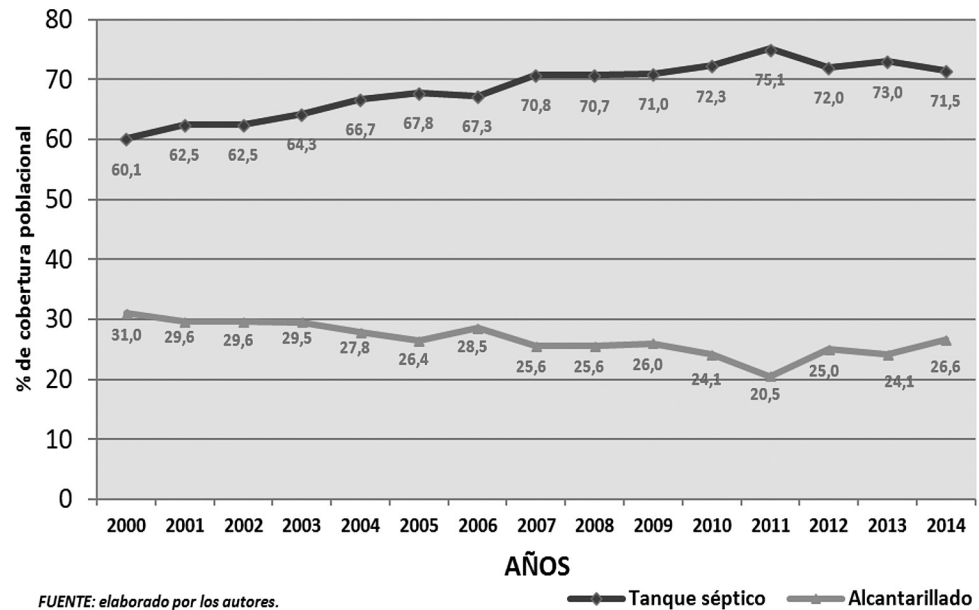

Figura 2. Análisis comparativo del uso de alcantarillado y tanques sépticos en Costa Rica: periodo 2000-2014 
Expectativas de saneamiento para el año 2021

El Proyecto de Mejoramiento Ambiental del Área Metropolitana fue aprobado mediante la Ley 8559, con un préstamo del Banco Japonés para la Cooperación Internacional (JBIC). En marzo de 2007 se estableció la Unidad Ejecutora AyA-JBIC con su equipo profesional, para entender el problema de saneamiento urbano en 11 cantones de San José. El costo de la obra para la Etapa 1 asciende a US\$345 millones.

Con este proyecto, se estima que como resultado de la rehabilitación y extensión del alcantarillado sanitario y la construcción de la Planta de Tratamiento de Los Tajos, la población cubierta pasará de un 45\% (700.000 habitantes) a un 65\% (1.070.000 habitantes), lo que indica que a nivel nacional las aguas de alcantarillado sin tratamiento disminuirán de 20,1\% a 0,5\%. Por el contrario, las aguas residuales con tratamiento, por medio de la nueva planta, pasarán de 4,5\% a 26,8\%. Este proyecto deberá estar terminado en su primera etapa en el año 2018, y uno de sus principales objetivos es "sanear" la cuenca Virilla-Tárcoles (Contraloría General de la República, 2011). No obstante, el proyecto no contempla la disminución de los desechos sólidos, los cuales, junto con las aguas residuales, son las principales causas del deterioro de esta cuenca.

En razón de esto, el Programa Bandera Azul Ecológica (Comisión Nacional del Programa Bandera Azul Ecológica, 2013) ha establecido el proyecto denominado "Disminución de los Desechos de la Cuenca Virilla-Tárcoles: 2013-2000" (Comisión Nacional del Programa Bandera Azul Ecológica, 2012), con el propósito de hacer una transformación cultural para educar a la población y así erradicar la costumbre de depositar los desechos sólidos en los cauces de los ríos que conforman esta cuenca.

\section{Analisis de resultados}

Para efectos prácticos, el análisis de los resultados se realiza en el mismo orden de aparición de los objetivos específicos.

\section{Infecciones e infestaciones relacionados con las excretas}

A través de la historia de la humanidad, el contacto con las excretas, o material fecal, ha sido la principal fuente de contagio de enfermedades infecciosas. Los factores involucrados con la transmisión de las diferentes patologías son la carga de excretas, la latencia, persistencia y multiplicación de los virus, bacterias, protozoarios y otros tipos de parásitos como los helmintos; además, depende de la dosis infecciosa y de las costumbres y fortaleza del aparato inmunológico de cada ser humano. Lógicamente, estos mismos aspectos participan en la transmisión de enfermedades entre los animales, el ser humano y los animales y viceversa.

En la transmisión de estas enfermedades vinculadas con las excretas entran en juego varios mecanismos de propagación, como el fecal-oral, el hídrico, el contacto con el suelo y con insectos. En razón del impacto de estas enfermedades infecciosas en el desarrollo de la humanidad, el manejo, tratamiento y disposición adecuada de las excretas ha sido un determinante esencial de la salud pública en las diferentes culturas del mundo, y sin lugar a dudas lo es o será en otros seres vivientes del planeta.

\section{Mecanismos de disposición de excretas}

La UNICEF y la OMS, con el propósito de medir los avances de los ODM en los diferentes países del mundo, estableció el concepto de "Instalaciones de Saneamiento Mejoradas", brindando el mismo peso a la disposición de las excretas, o aguas residuales domésticas, por alcantarillado con tratamiento, alcantarillado sin tratamiento o cloacas, tanques sépticos y los diferentes 
tipos de letrinas (de pozo mejorada con ventilación, con loza y al retrete de compostaje). Evidentemente, estos mecanismos permiten alejar la materia fecal de los hogares o viviendas, pero son muy desiguales para medir el desarrollo de los pueblos o las naciones.

Cobertura de la población con disposición de excretas

Los resultados de las coberturas por regiones de planificación, zona urbana o rural y la totalidad del país en saneamiento o DE, resumidos en el Cuadro 2, indican que:

- De los 4.772.098 habitantes de Costa Rica, en el año 2014, 3.412.099 tenían tanque séptico para disponer o tratar sus aguas residuales domésticas, lo que equivale al 71,5\%. Además 1.268.705 personas evacuaron sus excretas por medio de alcantarillado sanitario o cloacas, para un $26,6 \%$. Un 1,5\% de la población utilizó letrinas o excusado de hueco (73.462 habitantes) y 17.832 habitantes no contaban con ninguno de estos tres mecanismos, es decir, el 0,4\% utiliza el antiguo mecanismo de disponer sus excretas a cielo abierto. Estos datos se visualizan en la Figura 1, mediante un gráfico de pastel.

- En el área urbana, el 65,4\% usaba tanque séptico, en comparación con el 87,5\% en el área rural; con respecto al uso de alcantarillado, el 51,8\% se concentra en el área rural. El uso de letrinas se ubica en un 5,14\% en el área rural y un 0,4\% en la urbana. En el caso de la población que no tiene ningún servicio sanitario, llama la atención que 11.364 personas del área urbana $(0,32 \%)$ no cuentan con mecanismos adecuados de DE, contra 6.486 personas $(0,50 \%)$ en el área rural.

- La distribución de estos mecanismos de DE, en las seis regiones de planificación del país, demuestra que:

- La Región Central es la que presenta más uso de alcantarillado o cloacas, con un $35 \%$. Además, como es lógico, utiliza menos el tanque séptico, para un 64,3\%.

- Las regiones con más uso de tanque séptico son la Huetar Norte y la Brunca, con $87,6 \%$ y $87,4 \%$, respectivamente. Estas mismas regiones son también las que más utilizan el mecanismo "Cielo abierto", con 0,85\% y 0,84\%, respectivamente.

- Si aplicamos el concepto de "Instalaciones de Saneamiento Mejoradas" de la UNICEF y la OMS, paradójicamente Costa Rica se muestra como un país de avanzada en saneamiento, con un total estimado de 4.754.266 habitantes, o el 99,6\% con DE por alcantarillado, tanques sépticos o letrinas.

Sistemas de tratamiento de aguas residuales en Costa Rica

Inventario de sistemas convencionales

El LNA ha inventariado la eficiencia de seis lagunas de estabilización en Cañas, Liberia, Santa Cruz, Nicoya, Pérez Zeledón y Las Flores de Heredia, pertenecientes a AyA y la ESPH; además, 18 plantas de tratamiento, en su mayoría de lodos activados. Mención aparte merece la identificación y evaluación del único emisario submarino de Centroamérica, ubicado en la ciudad de Limón, inaugurado el 31 de octubre de 2004, con un costo total de US\$3.969.452,59. Por otro lado, aunque muchas personas no lo identifican como un sistema de tratamiento, los tanques sépticos usados por el $71,5 \%$ de la población son un sistema de tratamiento primario con una remoción de entre 30\% y 40\% de materia orgánica. Además, otros entes operadores, como los municipios y ASADAS tienen a su cargo 32 plantas de tratamiento. 
Eficiencia de la remoción de materia orgánica de los sistemas

de tratamiento inventariados y evaluados

Lagunas de estabilización del AyA

Los análisis y resultados promedios de las cinco lagunas de estabilización, cuatro de ellas ubicadas en Guanacaste y una en Pérez Zeledón, utilizando solo dos parámetros del Reglamento de Vertidos y Reúso de Aguas Residuales (Poder Ejecutivo, Costa Rica, 2007), Demanda Bioquímica de Oxígeno Total (BDO mg/L) y el número más probable de Coliformes fecales/100 mL (CF/100 mL) demuestran que:

- Ninguna laguna de estabilización, excepto Santa Cruz cumple con el valor máximo permitido de $50 \mathrm{mg} / \mathrm{L}$, oscilando entre $58 \mathrm{mg} / \mathrm{L}$ en Liberia y $100 \mathrm{mg} / \mathrm{L}$ de DBO total en Nicoya. Con respecto a la remoción de CF/100 mL, entre el agua cruda y tratada, los datos promedios van del $91 \%$ al $100 \%$. Sin embargo, el efluente que menos descarga $\mathrm{CF} / 100 \mathrm{~mL}$ al cuerpo receptor es la laguna de Cañas, con 67.585 de promedio de $\mathrm{CF} / 100 \mathrm{~mL}$, y la que más descarga es la laguna de Nicoya, con un promedio de 1.282.430 CF/100 mL.

- En el caso de los sistemas de tratamiento convencional de los otros 18 sistemas evaluados, los resultados promedio de DBO total en el agua tratada indican que 14 cumplen con el valor máximo permitido de $50 \mathrm{mg} / \mathrm{L}$ de DBO total. Dichos sistemas son Los Reyes, El Coyol y Villa Verano de Alajuela, Santa Cecilia de Puriscal, Boulevar Las Palamas, Rincón Verde II, Monte Lindo, André Chalé, Lomas de Pindeco, Don Edwin de Guápiles, Los Lagos, La Aurora, Real Santamaría Este y Real Santamaría Oeste de Heredia.

Con respecto a la descarga de $\mathrm{CF} / 100 \mathrm{~mL}$ en las aguas tratadas, es importante anotar lo siguiente:

a) La planta de El Roble de Puntarenas tiene un promedio de CF/100 mL de 56.651.624/100 $\mathrm{mL}$ en la descarga que va al estero de Puntarenas, con un porcentaje de eficiencia de -190\%; es decir, tiene mucho más contaminación fecal el efluente que la entrada de agua cruda de la planta.

b) Las plantas que menos descargan CF/100 mL son Lomas de Pindeco, con 100\% de remoción con $16.773 \mathrm{CF} / 100 \mathrm{~mL}$; André Chalé, con 100\% de remoción con 97.196 CF/100 mL; las Palmas (98\% de eficiencia) y 157.830 CF/100 mL; Don Edwin, con un promedio de CF/100 mL de 259.444 y Hacienda Los Reyes, con 242.379 CF/100 mL. Las restantes plantas descargan entre 981.870 CF/100 mL a 56.651.624 CF/100 mL.

c) En el caso de la eficiencia del Emisario Submarino de Limón, se observa una gran disminución en los 32 parámetros estudiados (ver cuadro 4).

Con respecto a los sistemas de tratamiento a cargo de otros entes operadores, cuatro de los cinco sistemas a cargo de la ESPH funcionaban adecuadamente en el año 2012. No se conoce la eficiencia de los sistemas a cargo de los municipios y ASADAS.

Evolución de las coberturas de disposición de excretas: 2000-2014

El saneamiento o disposición adecuada de excretas mediante alcantarillado y tanques sépticos en el periodo 2000-2014 en Costa Rica se ha fundamentado en el uso de tanques sépticos, evolucionando de un 60,1\% en el año 2000 a 75,1\% en 2011, para luego bajar levemente al $71,5 \%$ en 2014. Por otro lado, el uso de alcantarillado o cloaca alcanzó el 31,0\% en el año 2000, para disminuir a $20,5 \%$ en el 2011 y aumentar nuevamente a $26,6 \%$ en el 2014 . Este 
comportamiento se fundamenta en la implementación de pequeños sistemas de tratamiento en el Valle Central, la Región Brunca y Guápiles y algunas urbanizaciones.

\section{Expectativas de saneamiento hacia el año 2021 en Costa Rica}

El Proyecto de Mejoramiento Ambiental de San José tiene como propósito "mejorar" la salud ambiental de 11 cantones del Área Metropolitana, mediante la rehabilitación de aproximadamente $400 \mathrm{~km}$ de construcción de alcantarillado sanitario, y de una planta de tratamiento de lodos activados denominada "Los Tajos", que estará ubicada en La Uruca. La ejecución de la Etapa 1 de este proyecto permitirá aumentar de $22 \%$ a $28 \%$ en el tratamiento colectivo de aguas residuales en el año 2019. Por otro lado, con este y otros proyectos menores de alcantarillado sanitario en algunas zonas costeras (Jacó, Limón, Cieneguita y Puntarenas), se estima que la cobertura de la población con alcantarillado podría incrementarse al 53\%, mientras que el uso de tanques sépticos disminuiría de 71,5\% en 2014 a 48\% en 2021.

\section{Conclusiones y recomendaciones}

Los resultados obtenidos en las coberturas de DE en el año 2014, el análisis de la evolución en los últimos 15 años y las expectativas al año 2021 nos permiten hacer las siguientes conclusiones y recomendaciones.

\section{Conclusiones}

El uso del servicio sanitario y la disposición adecuada de excretas, aunado a la higiene y el lavado de manos, evitan la transmisión de enfermedades infecciosas vinculadas al contacto con excretas.

La UNICEF y la OMS, con el objetivo de medir los avances de los países en la Meta 7b de los ODM (1990-2015), establecieron el concepto de "Instalaciones de Saneamiento Mejoradas", en donde equivocadamente le dan el mismo peso al uso de alcantarillado con tratamiento, alcantarillado sin tratamiento o cloacas, al tanque séptico y a las letrinas. Esto impide medir el verdadero avance en el tratamiento y la adecuada DE en las naciones del mundo.

Los datos aportados por la Encuesta de Hogares del INEC indican que de 4.772.098 habitantes, 3.412.099 utilizaron el tanque séptico en el año 2014 (71,5\%), 1.268.705 usaron alcantarillado $(26,6 \%), 1,5 \%$ utilizan letrinas y 17.832 habitantes no tenían servicio sanitario o utilizaron la DE a cielo abierto.

El tanque séptico presentó mayor cobertura en la población rural (87,5\%), con respecto a la urbana (65,4\%). Las regiones con mayor uso de tanque séptico son la Huetar y la Brunca, con $87,6 \%$ y $87,4 \%$, respectivamente; estas mismas regiones son también las más que más usan el mecanismo a cielo abierto, con 0,85\% y 0,84\%, respectivamente.

El inventario de los sistemas de tratamiento controlados por el AyA suma 21, de los cuales cinco son lagunas de estabilización, 15 plantas de tratamiento y el emisario submarino ubicado en la ciudad de Limón, además de los que son operados por la EPSH y las municipalidades y ASADAS, para un total de 58 sistemas.

Del total de sistemas de tratamiento evaluados por el LNA, cinco lagunas no cumplen con el Reglamento de Vertidos y Reúso de Aguas Residuales, ${ }^{(24)}$ al menos en el parámetro de DBO total de $50 \mathrm{mg} / \mathrm{L}$ en la salida de las plantas. En cuanto al tratamiento convencional, 10 de las 14 plantas $(71 \%)$ cumplen con la legislación vigente. El emisario submarino ha sido muy eficiente en la remoción de la materia orgánica y los CF/100 mL en su zona de influencia. Los datos de la ESPH indican que cuatro de los sistemas cumplen con la reglamentación. Con respecto a los 
32 sistemas a cargo de los municipios y las ASADAS, no se tienen datos de sus eficiencias de remoción de materia orgánica e inorgánica.

La evolución de las coberturas de DE en el periodo 2000-2014 demuestra un favorecimiento de los tanques sépticos de entre 69\% y 71,5\%, alcanzando su uso máximo en 2011 con el 75,1\%. El uso de alcantarillado ha oscilado entre 31,0\% y 26,6\% en el periodo 2000-2014.

El análisis de la expectativa de los proyectos de alcantarillado sanitario con tratamiento para los años 2015 a 2021 permite estimar que el país podría alcanzar entre un 22\% a 28\% de tratamiento de las aguas residuales para 2019, siempre y cuando se concluya la Etapa 1 del Proyecto de Mejoramiento Ambiental de San José, que se encuentra en ejecución. Junto a esto, la construcción de otros proyectos en zonas costeras permitiría alcanzar un 53\% de cobertura de DE por alcantarillado y una disminución de la cobertura de la población con tanque séptico al $48 \%$ en el año 2021.

\section{Recomendaciones}

El AyA, la ESPH y las 81 municipalidades del país deben trabajar en la transformación cultural de la población, para erradicar la mala costumbre de depositar todos los desechos sólidos en las cuencas de los ríos. De no ser así, por ejemplo, el esfuerzo del proyecto de mejoramiento ambiental de San José, con la rehabilitación del alcantarillado y la planta de tratamiento de lodos activados, se verá opacado, debido a la presencia permanente de basura o todo tipo de desechos.

Además de la recomendación anterior, se debe fortalecer el proyecto de reducción de los desechos en la cuenca Virilla-Tárcoles, promovida por el Programa Bandera Azul Ecológica, para el periodo 2013-2100.

El AyA, como entidad rectora en agua potable y saneamiento, debe actualizar y poner en práctica el Programa Nacional de Manejo Adecuado de las Aguas Residuales, con sus ocho componentes, distribuidos en subprogramas, proyectos, acciones y metas.

El AyA, el Ministerio de Salud y otras entidades de gobierno deben concretizar la política de saneamiento para los próximos años.

\section{Bibliografía}

Araya, Á.; Barboza, R.; Ramírez, W; Rodríguez, A. (2009). Informe técnico sobre la inspección tramo fecal del Emisario de Limón. San José, Costa Rica; Instituto Costarricense de Acueductos y Alcantarillados.

Araya, D.; Araya, Á.; Trejos, S. (2003). Estudio sobre la situación de las Aguas Residuales de tipo ordinario en Costa Rica. San José, Costa Rica; OPS/OMS y AyA.

Artman, K; Chadwick, E. (2015). Cholera and the Thames. Disponible en: www.choleraandthethames.co.uk>Edwin

Instituto Costarricense de Acueductos y Alcantarillados (AyA). (2009). Programa Nacional de Manejo Adecuado de Aguas Residuales. San José, Costa Rica; AyA.

Baltazar, J; Solon, F. (1989). Disposal of faeces o children under two years old and diarrhea. Heller, L. (1997). Tomado de: Saneamiento y Salud. (1997). Brasil: Organización Panamericana de la Salud (OPS)/Organización Mundial de la Salud (OMS). 18 (4) (supp.2), 16-19.

Comisión Nacional del Programa Bandera Azul Ecológica. (2012). Proyecto de Disminución de los Desechos en la Cuenca Virilla-Tárcoles. Laboratorio Nacional de Aguas. Tres Ríos, La Unión, Cartago, Costa Rica.

Comisión Nacional del Programa Bandera Azul Ecológica. (2013). Plan Estratégico 2013-2017. Garabito, Puntarenas, Costa Rica.

Contraloría General de la República. (2011). DFOE-AE-IF-10. Proyecto de Mejoramiento Ambiental del Área Metropolitana de San José, Costa Rica. Disponible en www.asamblea.go.cr>DFOE-AE-IF-10... 
Heller, L. (1997). Saneamiento y Salud. (1997). Brasil: Organización Panamericana de la Salud (OPS)/Organización Mundial de la Salud (OMS).

Instituto Nacional de Estadística y Censos (INEC). (2001). Censo Nacional de Población y Vivienda 2000. Disponible en es.m.wikipedia.org>wiki >censo_costar

Instituto Nacional de Estadística y Censos (INEC). (2012). Censo Nacional de Población y Vivienda 2011. San José, Costa Rica. Disponible en es.m.wikipedia.org>wiki>censo_costar

Instituto Nacional de Estadística y Censos (INEC). (2014). Encuesta Nacional de Hogares 2013. San José, Costa Rica; INEC.

Jouravlev, A. (2004). Los servicios de agua potable y saneamiento en el umbral del siglo XXI. Documento en linea: http://google.co.cr/bitstream/handle/11362. Santiago de Chile; Naciones Unidas/CEPAL; junio, 2004.

Llopie, P.; Tynan, N. (2011). La historia del Saneamiento. Disponible en www.ungarbi-eu... >Drenaje Sostenible.

Mora, D. (1992). Evolución y Expectativas de la contaminación de la cuenca Virilla-Tárcoles. Revista Costarricense de Salud Pública, (2), 18-23.

Mora, D. (2003). Agua para Consumo Humano y disposición de Excretas en Costa Rica: situación actual y expectativa. Tecnología en Marcha, 16(3), 61-76.

Mora, D. (2005). Saneamiento, Educación y Salud. San José: Editorama.

Mora, D. (2009). Situación actual del agua para consumo humano y aguas residuales en Costa Rica- 1991. Revista Biocenosis, 2, 74-80.

Unidad Ejecutora AyA JBIC y Navarro, J. (2015). Panorama del Proyecto Mejoramiento Ambiental del Área Metropolitana de San José. Disponible en Busde.plaho.org>aya>navarro. San José, Costa Rica.

Pickover, C. (2012). El Libro de la Medicina. Madrid: Ilus Books. S.L. ISBN 978-90 8998-209-3.

Poder Ejecutivo, Costa Rica. (1997). Designación del Laboratorio Central de AyA como Laboratorio Nacional de Aguas. La Gaceta N¹00. Decreto Ejecutivo 26066-S.

Poder Ejecutivo, Costa Rica. (2007). Reglamento de Vertido y Reúso de Aguas Residuales. La Gaceta №55. Decreto Ejecutivo N³3601 Minae-S.

Programa Estado de La Nación. (2013). 19 Informe Estado de La Nación en Desarrollo Humano Sostenible. San José, Costra Rica; Programa Estado de la Nación.

Ramírez, J. \& Valiente, C. (2015). Informe Anual de Aguas Residuales. Laboratorio Nacional de Aguas. Tres Ríos, La Unión, Cartago.

Reynolds, K. (2015). Tratamiento de Aguas Residuales en Latinoamérica. Disponible en www.agualatinoamerica.com

Ruiz, F. (2012). Gestión de las Excretas y Aguas Residuales en Costa Rica. Situación y Perspectiva. San José: FOCARDS y Cooperación COSUDE.

Fondo de las Naciones Unidas par la Infancia (UNICEF)/Organización Mundial de la Salud (OMS) (2006). Progreso para la Infancia: un balance sobre agua y saneamiento. New York, USA.

Unidad Ejecutora AyA-JBIC. (2015). Proyecto de mejoramiento ambiental del Área Metropolitana tiene 10\% de avance. San José: Noticias Teletica Canal 7. Disponible en www.teletica.com>noticias. 